\title{
Few, Activity-Dependent, and Ubiquitous VGLUT1/VGAT Terminals in Rat and Mouse Brain
}

Giorgia Fattorini ${ }^{1,2 *}$, Chiara Ciriachi ${ }^{1 \dagger}$ and Fiorenzo Conti ${ }^{1,2,3}$

${ }^{1}$ Department of Experimental and Clinical Medicine, Section of Neuroscience and Cell Biology, Università Politecnica delle Marche, Ancona, Italy, ${ }^{2}$ Center for Neurobiology of Aging, Istituto Nazionale di Riposo e Cura per Anziani - Istituto di Ricovero e Cura a Carattere Scientifico, Ancona, Italy, ${ }^{3}$ Fondazione di Medicina Molecolare, Università Politecnica delle Marche, Ancona, Italy

In the neocortex of adult rats VGLUT1 and VGAT co-localize in axon terminals which form both symmetric and asymmetric synapses. They are expressed in the same synaptic vesicles which participate in the exo-endocytotic cycle. Virtually nothing, however, is known on whether VGLUT1NGAT co-localization occurs in other brain regions. We therefore mapped the distribution of terminals co-expressing VGLUT1NGAT in the striatum, hippocampus, thalamus, and cerebellar and cerebral cortices of rats and mice. Confocal microscopy analysis revealed that, in both rat and mouse brain, VGLUT1NGAT+ terminals were present in all brain regions studied, and that their percentage was low and comparable in both species. These results provide the first demonstration that co-expression of VGLUT1 and VGAT is a widespread phenomenon. Since VGLUT1NGAT+ axon terminals are regulated in an activity-dependent manner and co-release glutamate and GABA, we hypothesize that, though not numerous, they can contribute to regulating excitation/inhibition balance in physiological conditions, thereby playing a role in several neurological and psychiatric diseases.

Keywords: VGLUT1, VGAT, co-localization, E/I balance

\section{INTRODUCTION}

Glutamate and $\gamma$-aminobutyric acid (GABA) are the most important excitatory and inhibitory neurotransmitters in central nervous system, respectively (Conti and Weinberg, 1999; Cherubini and Conti, 2001). Replenishment of glutamatergic and GABAergic synaptic vesicles, a fundamental step in synaptic physiology, is mediated by specific vesicular transporters termed VGLUT1-3 (Gras et al., 2002; Fremeau et al., 2004; Takamori, 2006) and VGAT, respectively (McIntire et al., 1997; Sagne et al., 1997; Takamori et al., 2000).

Safiulina et al. (2006) reported that VGLUT1 and VGAT co-localize in developing hippocampal mossy fibers terminals. Subsequently, we demonstrated in rat adult neocortex that VGLUT1 and VGAT are co-localized in a subset of axon terminals which form both symmetric and asymmetric synapses, that they are sorted to the same vesicles, and that these vesicles participate in the exo-endocytotic cycle (Fattorini et al., 2009). More recently, we showed that glutamatergic and GABAergic responses can be recorded from rat cortical neurons in cultures, indicating the occurrence of glutamate and GABA co-release from neurons co-expressing VGLUT1 and VGAT (mixed synapses), and that the percentage of mixed synapses is regulated in an activity-dependent manner (Fattorini et al., 2015). 


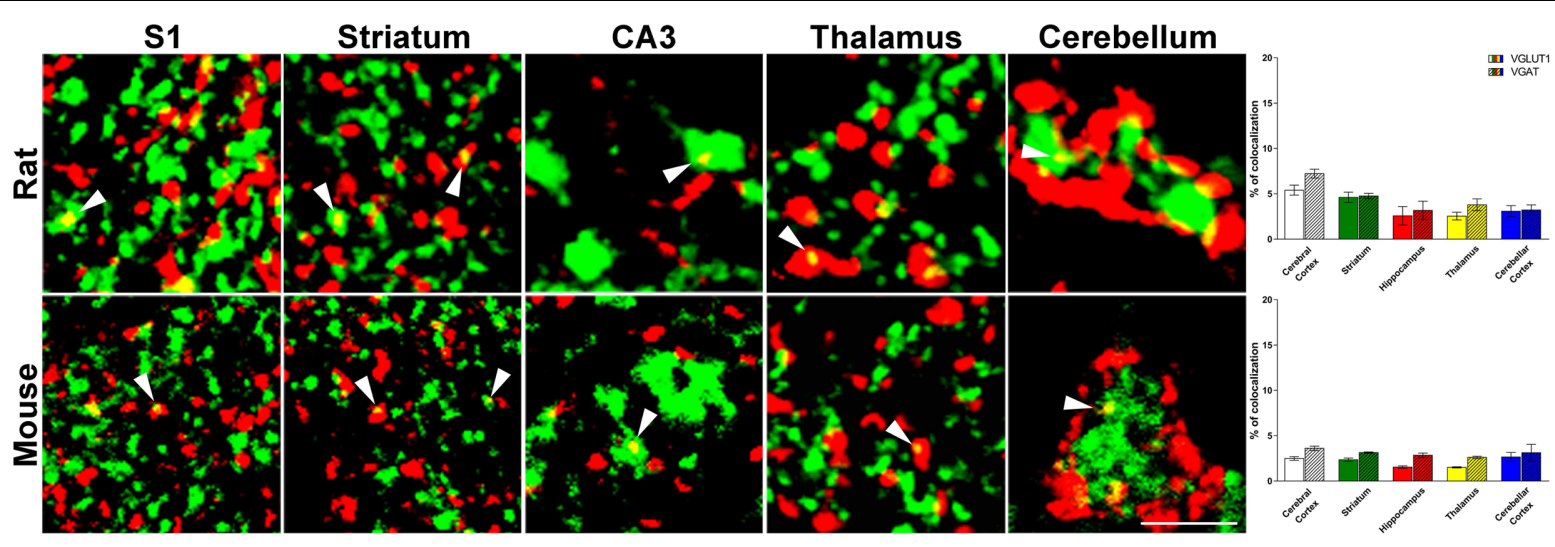

FIGURE 1 | Confocal analysis of rat and mouse brain VGLUT1(green)/VGAT+(red) terminals. Cerebral cortex: layers II-III of S1; Striatum: caudate-putamen (CPu) nucleus; Hippocampus: Stratum Lucidum of CA3; Thalamus: anterodorsal nucleus (AD); Cerebellar cortex: granular layers. Arrowheads point to example of co-localization between VGLUT1 and VGAT. After perfusion brains were removed stereotaxically in order to obtain the regions of interest (Paxinos and Watson, 1986; Paxinos and Franklin, 2001): -0.3 to $-6.8 \mathrm{~mm}$ from the bregma for rat brain; 0.0 to $-3.6 \mathrm{~mm}$ from the bregma for mouse brain. Sections were incubated for $1 \mathrm{~h}$ in newborn calf serum (NBCS; $10 \%$ in PB with $0.2 \%$ Triton X-100), and then for $2 \mathrm{~h}$ at room temperature plus overnight at $4^{\circ} \mathrm{C}$ in a solution containing a mixture of anti-VGAT (made in rabbit; Synaptic System, Germany, \#131003, RRID:AB_887869; 1:500 rat, 1:400 mouse) (Takamori et al., 2000) and, only in rat experiments, anti-VGLUT1 (made in guinea-pig; Millipore, Billerica, MA, United States, AB5905, RRID:AB_2301751; 1:1000) (Melone et al., 2005) primary antibodies, for mouse experiments we use fluorescent VGLUT1 (Venus) knock-in mouse (Herzog et al., 2011). The next day, sections were incubated in a mixture of CY3 (Jackson ImmunoResearch, West Grove, PA, United States, \#711-166-152; 1:250) and (only in rat experiments) Alexa-488 (Jackson Immunoresearch, West Grove, PA, United States, \#706-546-148; 1:250). Images were acquired using a Leica TCS-SP2 confocal laser microscope equipped with an argon (488 nm) and a helium/neon $(543 \mathrm{~nm})$ using a $\times 63$ oil immersion lens (numerical aperture 1.4; pinhole 1.0 and image size $512 \times 512$ pixels, yielding a pixel size of $0.155 \mu \mathrm{m}$ (rat) and $0.133 \mu \mathrm{m}$ (mouse) from a plane in which the resolution of both stains was optimal and never $>1.8 \mu \mathrm{m}$ from the surface (Melone et al., 2005). Green and red channels were acquired sequentially. To improve the signal/noise ratio, 10 frames/image were averaged. Images were deconvolved using Iterative Deconvolve 3D plugin (Dougherty, 2005) of ImageJ software (v. 1.48; NIH). Scale bars: $5 \mu \mathrm{m}$. Quantitative analysis of VGLUT1/VGAT co-localization in rat (up) and mouse (down) brain regions. The results show that the percentage of VGLUT1/NGAT+ terminals is comparable across regions and species. Three-four animals/regions. Data represent mean \pm S.E.M.

To date, very little is known on the occurrence of VGLUT1/VGAT co-localization in other brain regions, (see below). Based on their activity-dependent regulation, terminals co-expressing VGLUT1/VGAT appear suitable to control excitation-inhibition (E/I) balance; and on this basis we hypothesize that they may exhibit a widespread distribution in the brain. In order to test this hypothesis, we investigated the occurence of VGLUT1/VGAT terminals in different brain regions.

\section{CO-EXPRESSION OF VGLUT1 AND VGAT IS A WIDESPREAD PHENOMENON}

Confocal microscopy analysis revealed that in both rat and mouse brain VGLUT1/VGAT+ terminals were present in comparable amount in all brain regions studied (Figure $\mathbf{1}$ and Table 1).

In rat brain, the percentage of VGLUT1+ puncta coexpressing VGAT was $5.4 \pm 0.5 \%$ in cerebral cortex; $4.6 \pm 0.6 \%$ in striatum; $2.6 \pm 1.0 \%$ in hippocampus; $2.5 \pm 0.4 \%$ in thalamus; and $3.1 \pm 0.6 \%$ in cerebellar cortex, while the percentage of VGAT+ puncta co-expressing VGLUT1 was $6.0 \pm 0.5 \%$ in cerebral cortex; $4.8 \pm 0.3 \%$ in striatum; $3.2 \pm 1.0 \%$ in hippocampus; $3.8 \pm 0.7 \%$ in thalamus; and $3.2 \pm 0.6 \%$ in cerebellar cortex (Figure 1). Details on individual nuclei, layers or sub-regions are presented in Table $\mathbf{1 .}$
Mice are more widely used than rats for generating transgenic animals, an approach that could be useful in the future to define the functional role of the system of VGLUT1/VGAT co-expressing terminals; for this reason, we performed the same analysis in this species. In mouse brain, the percentage of VGLUT1+ puncta co-expressing VGAT was $2.5 \pm 0.2 \%$ in cerebral cortex; $2.4 \pm 0.2 \%$ in striatum; $1.6 \pm 0.1 \%$ in hippocampus; $1.5 \pm 0.07 \%$ in thalamus; and $2.7 \pm 0.5$ in cerebellar cortex, while the percentage of VGAT+ puncta co-expressing VGLUT1 was $3.6 \pm 0.2 \%$ in cerebral cortex; $3.1 \pm 0.07 \%$ in striatum; $2.8 \pm 0.2$ in hippocampus; $2.6 \pm 0.1$ in thalamus; and $3.1 \pm 0.9$ in cerebellar cortex (Figure 1). Details on individual nuclei, layers or sub-regions are given in Table 1.

\section{VGLUT1/VGAT TERMINALS MAY CONTRIBUTE TO E/I BALANCE IN A MULTITUDE OF BRAIN CIRCUITS}

In the last few years, several papers have documented that VGLUT1 and VGAT are co-expressed in cerebral cortex, hippocampus and cerebellum of rats and mice (Gutierrez, 2005; Safiulina et al., 2006; Uchigashima et al., 2007; Fattorini et al., 2009; Zander et al., 2010; Beltran and Gutierrez, 2012). To date, the occurrence of VGLUT1/VGAT co-expression in other brain regions of both species is poorly understood. Here, we showed for the first time that co-expression of VGLUT1 and VGAT is a 
TABLE 1 | VGLUT1-VGAT colocalization in rat and mouse brain subregions.

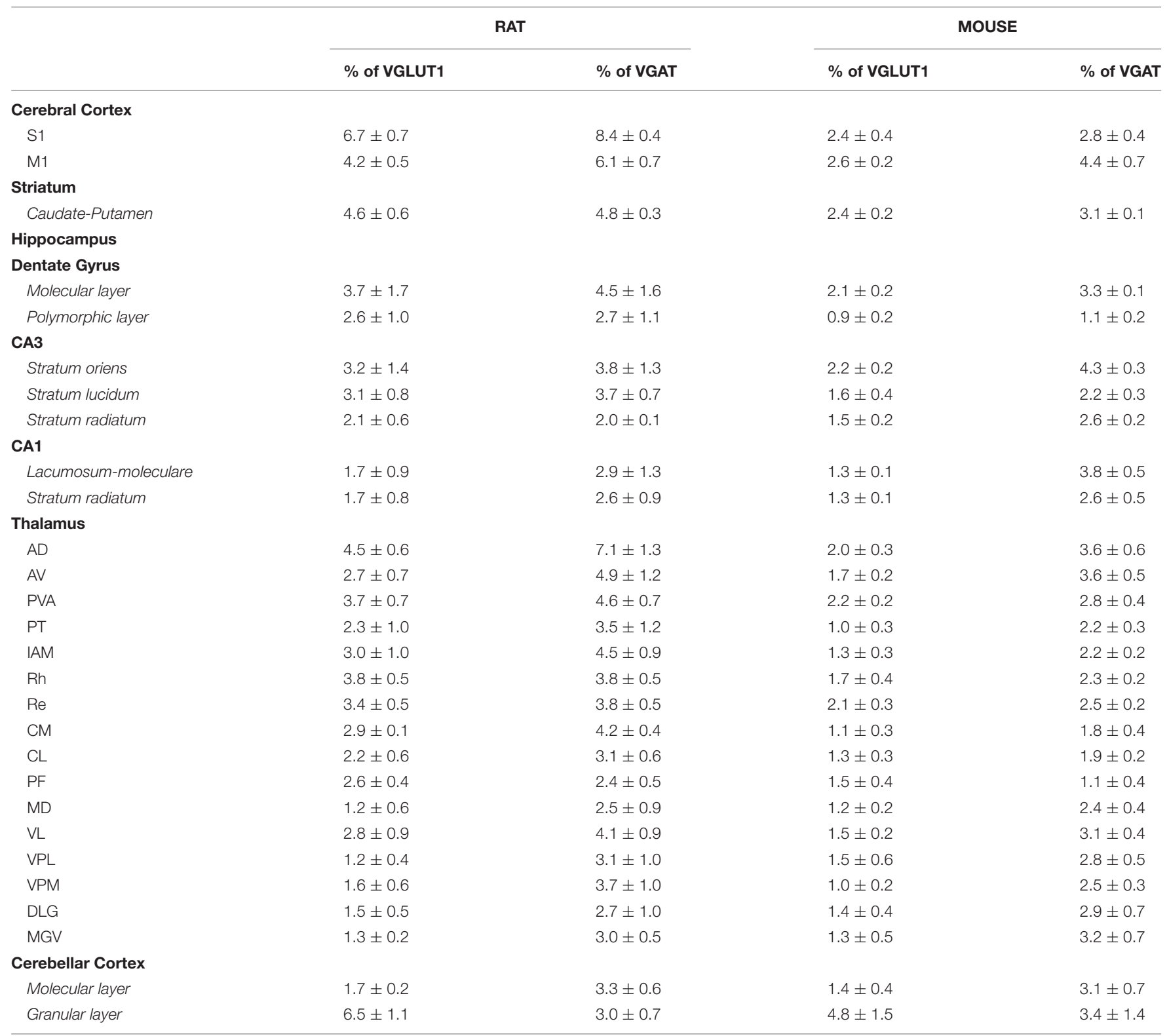

Cerebral cortex: layers II-III of S1 and M1 (six fields/section; rat: one section/animal, four rats; mouse: two sections/animal, three mice); Striatum: caudate-putamen (CPu) nucleus (six fields/section, two section/animal, three animals); Hippocampus: Molecular layer and Polymorphic cell layer of the Dentate Gyrus; Stratum Oriens, Stratum Lucidum and Stratum Radiatum of CA3; Stratum Lacunosum-Moleculare and Stratum Radiatum of CA1 (three fields/layer/section, two sections/animal, three animals); Thalamus: anterior nuclear group [anterodorsal nucleus (AD), anteroventral nucleus (AV)]; dorsomedial nucleus (MD); midline nuclear group [paratenial nucleus (PT), paraventricular nucleus (PVA), interanteromedial nucleus (IAM), reuniens nucleus (Re), rhomboidal nucleus (Rh)]; intralaminar nuclei [central lateral nucleus (CL), central medial nucleus (CM), parafascicular nucleus (PF)]; ventral nuclear group [ventral lateral nucleus (VL), ventral posterolateral (VPL), ventral posteromedial (VPM)]; medial geniculate complex [medial geniculate nucleus (MGV)]; dorsal lateral geniculate nucleus (DLG; three fields/nucleus/section, two sections/animal, three animals); Cerebellum: Molecular and granular layers of cortex (six fields/layer/section, two sections/animal, three animals). Data represent mean \pm S.E.M.

widespread phenomenon, and that the co-localization of the two vesicular transporters is not markedly different between the two species, an observation that might be of some help for studying the role of VGLUT1/VGAT co-expression in animal models of neurological and psychiatric diseases.

The main data emerging from this study is that VGLUT1/VGAT+ terminals are present in all the anatomical structures studied of both species. The percentage of
VGLUT1/VGAT+ terminals is regulated in an activitydependent manner: reducing the activity of the neuronal network by addition of glutamate receptor antagonists to the cultures decreases the percentage of mixed synapses, whereas reducing spontaneous inhibition with bicuculline increases them (Fattorini et al., 2015). These findings support the idea that activity-dependent regulation of glutamate and GABA co-release might play a role in regulating E/I balance in cortical 
microcircuits, thereby contributing to regulate brain function in normal and pathological conditions. The present demonstration that VGLUT1/VGAT co-expression occurs in all brain regions studied suggests that these terminals may contribute to adjust $\mathrm{E} / \mathrm{I}$ balance in a multitude of brain circuits. For example, it is well known that an abnormal increase of glutamatergic and/or an abnormal decrease of GABAergic transmission in hippocampus promotes neuronal hyperexcitability and hypersynchronization, and may sustain epileptic networks (e.g., Bonansco and Fuenzalida, 2016). The present evidence of a population of hippocampal VGLUT1/VGAT+ axon terminals, demonstrated here for the first time in adult normal animals, raises the possibility that their dysregulation may generate E/I imbalance. In addition, controlling inhibitory and excitatory sources (both local and extrinsic) in higher-order thalamic nuclei may contribute to thalamic oscillations (Fogerson and Huguenard, 2016), implying that VGLUT1/VGAT + terminals-mediated E/I imbalance in these circuits may play a crucial role in some forms of epilepsy. Finally, the network of parvalbumin + fastspiking interneurons has attracted much interest in autism spectrum disorders (ASDs), and parvalbumin knockout mice exhibit behavioral phenotypes resembling core symptoms of the diseases. In the striatum of these mice, E/I balance is altered by modification of both inhibitory and excitatory synaptic transmission, leading to the hypothesis that downregulation of parvalbumin might be central to the neurobiological basis of the diseases (Wohr et al., 2015). In this context, it is worth noting that in fast-spiking-enriched cultures the percentage of VGLUT1/VGAT + terminals is increased compared to controls (Fattorini et al., 2015), thus making it conceivable that VGLUT1/VGAT+ terminals in striatum (as well as in other brain regions) may play a role.

\section{CONCLUSION}

We showed that VGLUT1/VGAT+ axon terminals are present in all structures studied in both rat and mouse

\section{REFERENCES}

Beltran, J. Q., and Gutierrez, R. (2012). Co-release of glutamate and GABA from single, identified mossy fibre giant boutons. J. Physiol. 590, 4789-4800. doi: 10.1113/jphysiol.2012.236372

Bonansco, C., and Fuenzalida, M. (2016). Plasticity of hippocampal excitatoryinhibitory balance: missing the synaptic control in the epileptic brain. Neural Plast. 2016:8607038. doi: 10.1155/2016/8607038

Cherubini, E., and Conti, F. (2001). Generating diversity at GABAergic synapses. Trends Neurosci. 24, 155-162. doi: 10.1016/S0166-2236(00)01724-0

Conti, F., and Weinberg, R. J. (1999). Shaping excitation at glutamatergic synapses. Trends Neurosci. 22, 451-458. doi: 10.1016/S0166-2236(99)01445-9

Dougherty, R. P. (2005). "Extensions of DAMAS and benefits and limitations of deconvolution in beamforming," in Proceedings of the 11th AIAA/CEAS Aeroacoustics Conference, (Monterey, CA: American Institute of Aeronautics and Astronautics). doi: 10.2514/6.2005-2961

Fattorini, G., Antonucci, F., Menna, E., Matteoli, M., and Conti, F. (2015). Coexpression of VGLUT1 and VGAT sustains glutamate and GABA co-release and is regulated by activity in cortical neurons. J. Cell Sci. 128, 1669-1673. doi: $10.1242 /$ jcs. 164210 brain. Their percentage is relatively low, but given their activity-dependent regulation, it is conceivable that they may play a role in both physiological regulation of $\mathrm{E} / \mathrm{I}$ and in the pathophysiology of several neurological and psychiatric diseases.

\section{ETHICS STATEMENT}

All experimental procedures involving animals and their care were carried out in accordance with National laws and policies (D.L.26, March 14, 2014), and with the European Community Council Directive guidelines (2010/63/UE); all procedures were approved by the local authority veterinary services (Università Politecnica delle Marche).

\section{AUTHOR CONTRIBUTIONS}

GF developed the concept, analyzed the data, and wrote the manuscript. CC performed the experiments and analyzed the data. FC developed the concept and wrote the manuscript.

\section{FUNDING}

This work was made possible by grants provided by Ministero dell'Istruzione, dell'Università e della Ricerca (PRIN grants 2010JFYFY2 and 2015H4K2CR - LS5) to FC, and by Università Politecnica delle Marche to GF and FC.

\section{ACKNOWLEDGMENTS}

We thank Dr. Etienne Herzog, Interdisciplinary Institute for Neuroscience, Université de Bordeaux for generously sharing the VGLUT1 (Venus) knock-in mouse.

Fattorini, G., Verderio, C., Melone, M., Giovedi, S., Benfenati, F., Matteoli, M., et al. (2009). VGLUT1 and VGAT are sorted to the same population of synaptic vesicles in subsets of cortical axon terminals. J. Neurochem. 110, 1538-1546. doi: 10.1111/j.1471-4159.2009.06251.x

Fogerson, P. M., and Huguenard, J. R. (2016). Tapping the brakes: cellular and synaptic mechanisms that regulate thalamic oscillations. Neuron 92, 687-704. doi: 10.1016/j.neuron.2016.10.024

Fremeau, R. T. Jr., Voglmaier, S., Seal, R. P., and Edwards, R. H. (2004). VGLUTs define subsets of excitatory neurons and suggest novel roles for glutamate. Trends Neurosci. 27, 98-103. doi: 10.1016/j.tins.2003.11.005

Gras, C., Herzog, E., Bellenchi, G. C., Bernard, V., Ravassard, P., Pohl, M., et al. (2002). A third vesicular glutamate transporter expressed by cholinergic and serotoninergic neurons. J. Neurosci. 22, 5442-5451.

Gutierrez, R. (2005). The dual glutamatergic-GABAergic phenotype of hippocampal granule cells. Trends Neurosci. 28, 297-303. doi: 10.1016/j.tins.2005.04.005

Herzog, E., Nadrigny, F., Silm, K., Biesemann, C., Helling, I., Bersot, T., et al. (2011). In vivo imaging of intersynaptic vesicle exchange using VGLUT1 Venus knock-in mice. J Neurosci. 31, 15544-15559. doi: 10.1523/JNEUROSCI.207311.2011 
McIntire, S. L., Reimer, R. J., Schuske, K., Edwards, R. H., and Jorgensen, E. M. (1997). Identification and characterization of the vesicular GABA transporter. Nature 389, 870-876. doi: 10.1038/39908

Melone, M., Burette, A., and Weinberg, R. J. (2005). Light microscopic identification and immunocytochemical characterization of glutamatergic synapses in brain sections. J. Comp. Neurol. 492, 495-509. doi: 10.1002/cne. 20743

Paxinos, G., and Franklin, K. B. J. (2001). The Mouse Brain in Stereotaxic Coordinates. San Diego, CA: Academic Press.

Paxinos, G., and Watson, C. (1986). The Rat Brain in Stereotaxic Coordinates. New York, NY: Academic Press.

Safiulina, V. F., Fattorini, G., Conti, F., and Cherubini, E. (2006). GABAergic signaling at mossy fiber synapses in neonatal rat hippocampus. J. Neurosci. 26, 597-608. doi: 10.1523/JNEUROSCI.4493-05.2006

Sagne, C., El Mestikawy, S., Isambert, M. F., Hamon, M., Henry, J. P., Giros, B., et al. (1997). Cloning of a functional vesicular GABA and glycine transporter by screening of genome databases. FEBS Lett. 417, 177-183. doi: 10.1016/S00145793(97)01279-9

Takamori, S. (2006). VGLUTs: 'exciting' times for glutamatergic research? Neurosci. Res. 55, 343-351. doi: 10.1016/j.neures.2006.04.016

Takamori, S., Rhee, J. S., Rosenmund, C., and Jahn, R. (2000). Identification of a vesicular glutamate transporter that defines a glutamatergic phenotype in neurons. Nature 407, 189-194. doi: 10.1038/35025070
Uchigashima, M., Fukaya, M., Watanabe, M., and Kamiya, H. (2007) Evidence against GABA release from glutamatergic mossy fiber terminals in the developing hippocampus. J. Neurosci. 27, 8088-8100. doi: 10.1523/ JNEUROSCI.0702-07.2007

Wohr, M., Orduz, D., Gregory, P., Moreno, H., Khan, U., Vorckel, K. J., et al. (2015). Lack of parvalbumin in mice leads to behavioral deficits relevant to all human autism core symptoms and related neural morphofunctional abnormalities. Transl. Psychiatry 5:e525. doi: 10.1038/tp.2015.19

Zander, J. F., Munster-Wandowski, A., Brunk, I., Pahner, I., Gomez-Lira, G. Heinemann, U., et al. (2010). Synaptic and vesicular coexistence of VGLUT and VGAT in selected excitatory and inhibitory synapses. J. Neurosci. 30, 7634-7645. doi: 10.1523/JNEUROSCI.0141-10.2010

Conflict of Interest Statement: The authors declare that the research was conducted in the absence of any commercial or financial relationships that could be construed as a potential conflict of interest.

Copyright (c) 2017 Fattorini, Ciriachi and Conti. This is an open-access article distributed under the terms of the Creative Commons Attribution License (CC BY). The use, distribution or reproduction in other forums is permitted, provided the original author(s) or licensor are credited and that the original publication in this journal is cited, in accordance with accepted academic practice. No use, distribution or reproduction is permitted which does not comply with these terms. 